\title{
Chest wall hernia induced by high intensity focused ultrasound treatment of unresectable massive hepatocellular carcinoma: A case report
}

\author{
QI-WEN CHEN ${ }^{1,2 *}$, WEN-JING TENG ${ }^{3}$ and QIAN CHEN ${ }^{4}$
}

${ }^{1}$ Department of Integrated Oncology, Fudan University Shanghai Cancer Center, Shanghai 200032; ${ }^{2}$ Department of Oncology, Shanghai Medical College, Fudan University, Shanghai 200032; ${ }^{3}$ Cancer Center,

Shanghai Municipal Hospital of Traditional Chinese Medicine, Shanghai 200041;

${ }^{4}$ Department of Obstetrics of Chongqing Haifu Hospital, Non-invasive and Minimally Invasive Therapeutic

Research Center for Uterine Benign Diseases of Chongqing, Chongqing 401121, P.R. China

Received January 24, 2015; Accepted February 11, 2016

DOI: $10.3892 / \mathrm{ol} .2016 .4618$

\begin{abstract}
In the present study, a case of unresectable massive hepatocellular carcinoma (HCC) treated with high intensity focused ultrasound (HIFU) alone is reported. Although the treatment induced chest wall hernia, its efficacy in treating the HCC was demonstrated. The medical records of a patient with an unresectable massive tumor that was effectively treated with serial HIFU ablation were retrospectively studied. Chest wall hernia was detected as a complication of the HIFU treatment, which has not been reported thus far in the literature. The patient has survived for 44 months since the first diagnosis in September 2010. Treatment resulted in partial remission of the tumor, pain relief, decreased levels of alpha-fetoprotein and chest wall hernia, as a complication. Therefore, HIFU may be an effective approach for the treatment of unresectable HCC, although it may occasionally cause complications.
\end{abstract}

\section{Introduction}

Hepatocellular carcinoma (HCC) is one of the most common and lethal tumors worldwide (1). HCC is likely to be diagnosed at an advanced stage, when the liver function of the patients is poor (2). As a result, the majority of patients with advanced stage HCC do not fulfill the indications for surgical resection (3). The

Correspondence to: Professor Qian Chen, Department of Obstetrics of Chonqing Haifu Hospital, Non-Invasive and Minimally Invasive Therapeutic Research Center for Uterine Benign Diseases of Chongqing, 1 Renhe Qingsong Road, Chongqing 401121, P.R. China

E-mail: cqwly@hotmail.com

*Contributed equally

Key words: high intensity focused ultrasound, hepatocellular carcinoma, unresectable massive tumor utilization of transcatheter arterial chemoembolization (TACE), targeted drugs and radiofrequency ablation (RFA) for the treatment of HCC has achieved good clinical outcomes (4). However, these treatments present a number of limitations, including tumor location, size, blood supply and economical reasons (5).

High intensity focused ultrasound (HIFU) is a newly emerged local thermal ablation therapy that is characterized by causing minor trauma to patients and enabling a fast recovery (6). In addition, HIFU enables three-dimensional conformal treatment of solid tumors and enhances the body's immune response against the tumor (7). In the present study, a representative case of unresectable massive HCC treated successfully with HIFU alone is reported. Although the treatment induced a chest wall hernia, a satisfying clinical result was observed following HIFU therapy.

\section{Case report}

The patient was a 57-year-old male who was admitted to the Department of Integrated Oncology of Fudan University Shanghai Cancer Center (Shanghai, China) in September 2010 with complaints of occasional abdominal distension that had lasted for 2 years. The patient did not present with jaundice, abdominal pain, nausea or vomiting upon initial admission.

The medical history indicated that the patient had suffered hepatitis B for 30 years without receiving any anti-viral treatment. In September 2010, the patient was subjected to an abdominal computed tomography (CT) scan, which revealed a mass in the right lobe of the liver (which was suspected to be HCC with intrahepatic metastasis), in addition to cirrhosis, splenomegaly and portal hypertension. Blood test demonstrated $\alpha$-fetoprotein (AFP) levels of 3,200 $\mathrm{ng} / \mathrm{ml}$ (normal range, 0-10 $\mathrm{ng} / \mathrm{ml}$ ). Subsequently, the patient underwent fine needle aspiration of his hepatic lesion. The cytomorphological findings revealed the presence of crowded and giant tumor cells with bizarre shape and high nuclear-cytoplasmic ratio. The presence of an endothelial cell lining is one of the histopathological features indicative of HCC (Fig. 1). In consequence, the patient was diagnosed with 
HCC stage B, according to the Barcelona clinic liver cancer staging classification of HCC (8). Upon obtaining signed written informed consent, the patient was treated with hepatic arterial infusion chemotherapy with $150 \mathrm{mg}$ oxaliplatin and $1 \mathrm{~g}$ 5-fluorouracil. The patient did not receive embolization with lipiodol, since no complete obstruction of the hepatic arterioportal fistula detected during the angiography was achieved. Following the procedure, the patient experienced grade 3 hyperemesis, according to the Common Terminology Criteria for Adverse Events published by the National Cancer Institute (Bethesda, MD, USA) (9). The patient was discharged upon receiving supportive care for 4 days.

In December 2010, the patient was readmitted into hospital due to elevated AFP levels $(5,732 \mathrm{ng} / \mathrm{ml})$. During the second admission, the patient refused to receive another hepatic arterial infusion chemotherapy, due to the previous hyperemesis. Treatment with sorafenib was then suggested to the patient, but it was declined due to financial reasons. In consequence, HIFU therapy was proposed to the patient, and written informed consent was obtained from the patient for the HIFU procedure.

Admission examination. The results of the biochemical analysis at the time of readmission were as follows: White blood cell count, $6.0 \times 10^{9}$ cells $/ 1$ (normal range, $3.5-9.5 \times 10^{9}$ cells $/ 1$ ); percentage of neutrophils, 66.5\% (normal range, 40-75\%); hemoglobin levels, $140 \mathrm{~g} / 1$ (normal range, 115-150 g/l); platelet count, $67 \times 10^{9}$ cells $/ 1$ (normal range, $125-350 \times 10^{9}$ cells $/ 1$ ); albumin levels, $41 \mathrm{~g} / 1$ (normal range, $40-55 \mathrm{~g} / \mathrm{l}$ ); total bilirubin levels, $26 \mu \mathrm{mol} / 1$ (normal range, 3.4-17.1 $\mu \mathrm{mol} / \mathrm{l}$ ); direct bilirubin levels, $8.6 \mu \mathrm{mol} / 1$ (normal range, $0-3.4 \mu \mathrm{mol} / 1$ ); alanine aminotransferase levels, $83 \mathrm{U} / 1$ (normal range, 7-40 U/1); aspartate aminotransferase levels, $76 \mathrm{U} / 1$ (normal range, 13-35 U/1); blood urea nitrogen levels, $6.3 \mathrm{mmol} / 1$ (normal range, 2.6-7.5 mmol/l); and creatinine levels, $68.6 \mu \mathrm{mol} / 1$ (normal range, $41-73 \mu \mathrm{mol} / \mathrm{l}$ ). Electrocardiogram did not report any abnormalities. Enhanced abdominal CT revealed a mass located in the right lobe of the liver with multiple intrahepatic metastases, liver cirrhosis, splenomegaly and portal hypertension (Fig. 2A-C).

Therapeutic range of HIFU treatment. The therapeutic range of HIFU treatment for the lesion covered the neoplastic lesion and extended to the area of normal tissue located within 2 .0-cm distance from the lesion.

HIFU treatment parameters and procedure. The procedure was completed under the guidance of real-time ultrasound using the JC-HIFU system (Chongqing Haifu-HIFU-Tech, Chongqing, China). The treatment parameters were as follows: Frequency, $0.85 \mathrm{MHz}$; focal diameter, $3 \mathrm{~mm}$; focal field length, $8 \mathrm{~mm}$; focal length, $151 \mathrm{~mm}$; and sound therapy peak power, $400 \mathrm{~W}$. The patient was placed in the right lateral position with full contact of the skin in the degassed water. Following anesthesia, sedation and immobilization of the patient, ultrasound (frequency, 2.5 MGz) was applied to identify the location, size and boundary of the lesion, in order to determine the treatment target of the tumor. Under ultrasound guidance and monitored in real time, the lesion was gradually ablated three-dimensionally to achieve a complete ablation of the entire tumor in order to avoid any residual malignancy. After the whole lesion was ablated thoroughly, the tumor tissue underwent coagulation

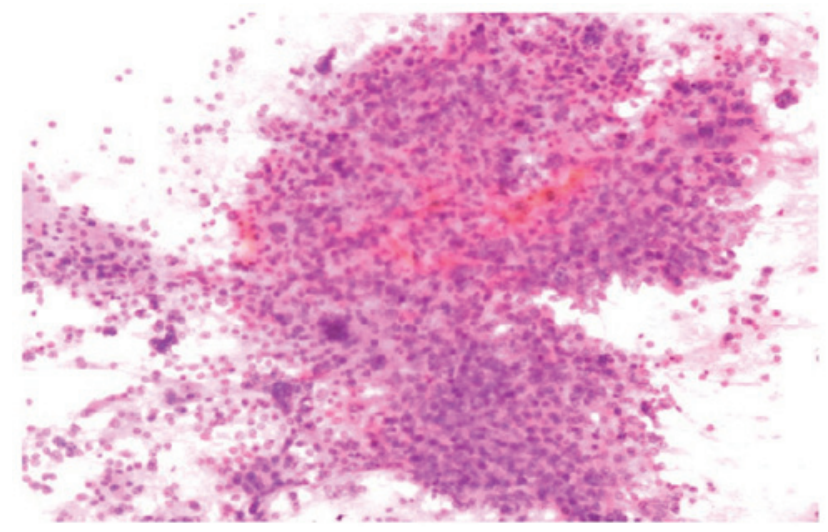

Figure 1. Crowded and giant tumor cells with bizarre shape and high nuclear-cytoplasmic ratio are observed in the tumor specimen. Anomalous cells that include vascular endothelial-like cells is one of the hallmark histopathological features indicative of hepatocellular carcinoma.

necrosis and exhibited a marked change of increased echo reflectance (Fig. 2E). Each slice of the targeted lesion was carefully and completely ablated from the deepest to the shallowest regions of the tumor, with the following parameters: Treatment power, $400 \mathrm{~W}$; line scan, $3 \mathrm{~mm} / \mathrm{sec}$; scanning distance, $5 \mathrm{~mm}$; number of irradiations, 3; treatment length, $4 \mathrm{~h}$; irradiation time, 4,850 sec; treatment intensity, $1,212 \mathrm{~kJ}$; total energy therapy, 1,940,000 J; and treatment volume, $11.43 \mathrm{~cm}^{3}$.

Post-operation treatment and follow-up information. Following HIFU treatment, the patient presented with swelling of the skin on his right chest. In consequence, an ice pack was applied intermittently for $2 \mathrm{~h}$, while liver protective and infection preventive intravenous drips were also administered to the patient, until the swelling of the regional skin gradually disappeared. Following discharge from the hospital, the patient noticed a reducible mass on his right chest wall 6 months later (Fig. 3A). The mass protruded outward while exhaling, and flattened upon massaging and inhaling (Fig. 3B). However, no treatment was received for this symptom, as it did not affect the patient's quality of life or cause any discomfort. In May 2014, abdominal CT scan (Fig. 3C) reported a tumor mass measuring $40 \times 45 \times 40 \mathrm{~mm}$ located in the right lobe of the liver, which was significantly reduced in size compared with the previous follow-up. The patient's chest X-ray (Fig. 3D) suggested right pleural effusion, right rib bone interruption and bone sclerotin discontinuation. Ultrasonography of the chest wall mass (Fig. 3E) revealed a hernia in the subcutaneous tissue. At the time of the last follow-up, conducted on May 2014, the patient had survived for 44 months since the diagnosis of HCC. The chest wall hernia had no effect on the quality of life of the patient, whose levels of AFP in serum had reduced to $182 \mathrm{ng} / \mathrm{ml}$.

\section{Discussion}

Surgery is the most effective treatment for HCC (10). However, the majority of cases of HCC are diagnosed at a late stage, resulting in the majority of patients unable to receive surgical resection (11). TACE has been used for the treatment of HCC, and achieves its therapeutic effect by utilizing lipiodol for the embolization of the major arterial supply to the liver tumor, 


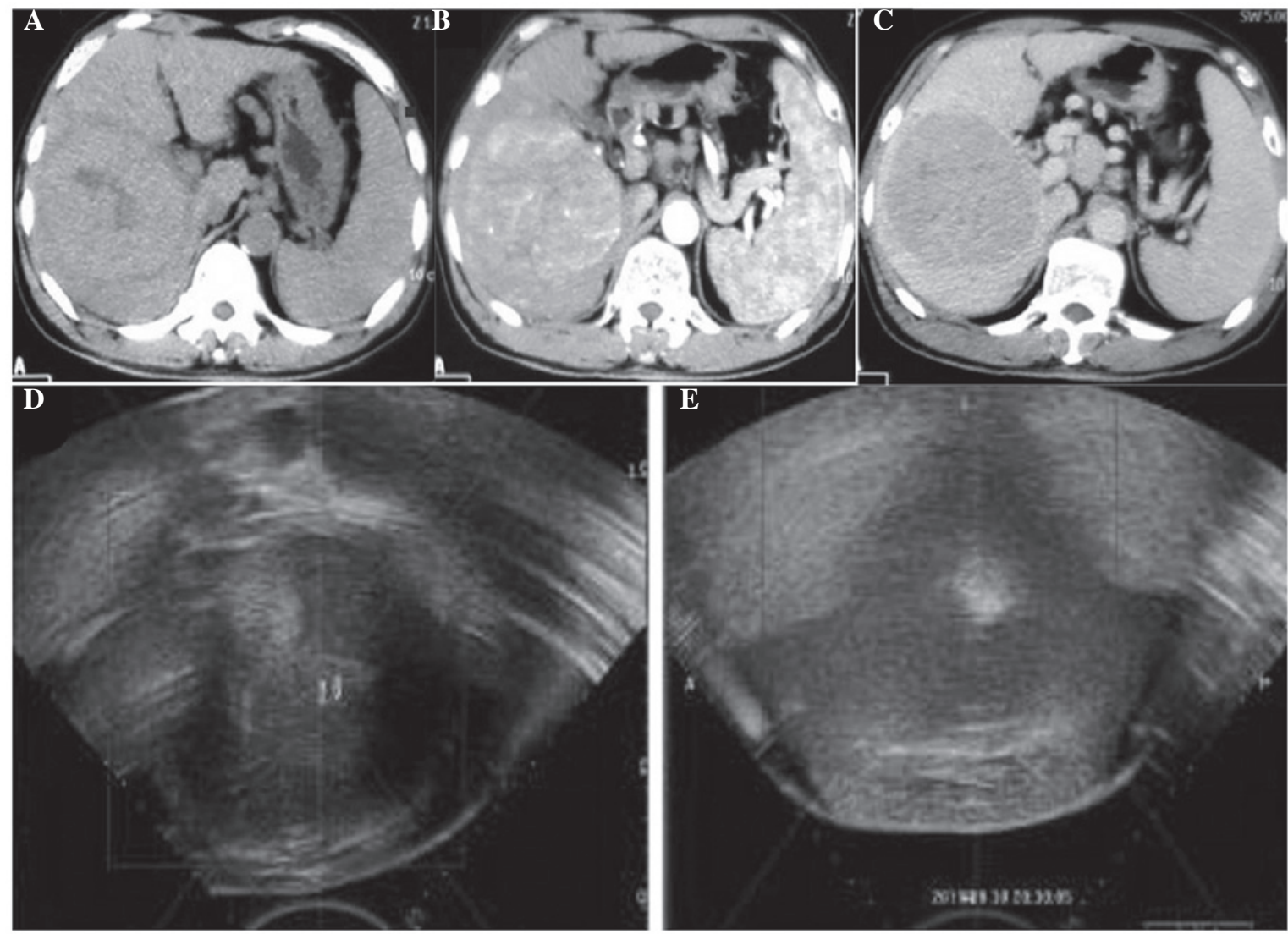

Figure 2. (A-C) Enhanced abdominal computed tomography revealed a mass in the right lobe of the liver with multiple intrahepatic metastases, liver cirrhosis, splenomegaly and portal hypertension. (A) Isodense mass on plain scanning; (B) Marked enhancement in teh arterial phase; and (C) Contrast agent washout in the delayed phase. Ultrasound image (D) prior to and (E) subsequent to high intensity focused ultrasound treatment. Following treatment, the tumor tissue underwent coagulation necrosis, and appeared as an obvious lumpy gray change in the ultrasound image.

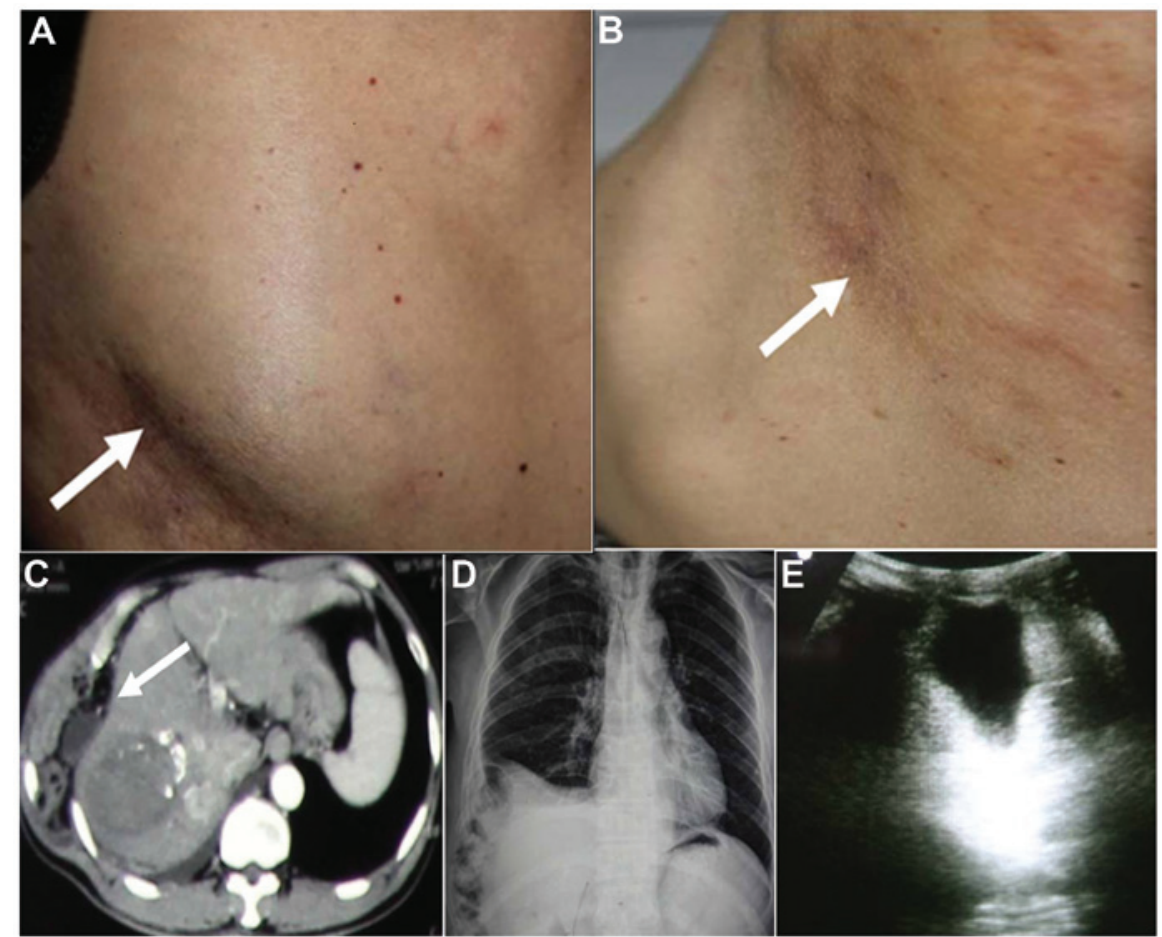

Figure 3. (A) Upon being discharged from the hospital, the patient noticed a reducible mass on his right chest wall 6 months later. (B) The mass protruded outward while exhaling, and flattened upon massaging and inhaling. (C) Abdominal computed tomography scan revealed a mass of $40 \times 45 \times 40 \mathrm{~mm}$ in size located in the right lobe of the liver, suggesting hepatocellular carcinoma with intrahepatic metastasis, cirrhosis and portal hypertension. (D) Chest X-ray suggested right pleural effusion, right rib bone interruption and bone sclerotin discontinuation. (E) Ultrasound of the chest wall mass demonstrated reduced chest wall subcutaneous tissue, possibly due to a chest wall hernia. 
thus inducing ischemic necrosis of the tumor (12). However, the present patient did not undergo lipiodol embolization, due to the inability of achieving complete obstruction of the hepatic arterioportal fistula. RFA is also useful for the treatment of HCC (13). However, the size of the tumor in the present case was too large to undergo RFA. The cost of targeted therapy for HCC in China is relatively high, thus the majority of Chinese patients are unable to afford it. Therefore, an effective modality with acceptable financial cost for the treatment of $\mathrm{HCC}$ is required.

HIFU is a relatively novel emerged local thermal ablation modality (14). By using local high temperature produced by ultrasound focusing on the targeted lesion, the temperature at the targeted lesion site rapidly rises to $65-100^{\circ} \mathrm{C}$, which induces coagulation necrosis in the lesion (15). HIFU achieves its three-dimensional conformal ablation and completely resects the lesion by moving the ultrasound applicator in order to vary the focused region inside the body (16). Via effective ablation, the tumor tissue undergoes coagulation necrosis and loss of replication, infiltration and metastasis capacity, eventually becoming fibrous degenerated or calcified tissue. In addition, multidrug resistant cancer cells and those in $\mathrm{G} 0$ phase are also sensitive to HIFU treatment. Furthermore, HIFU is able to stimulate the body's anti-tumor immune response subsequent to the procedure (17). HIFU is considered a superior treatment in terms of controlling tumor progression and prolonging survival, due to its advantages, which include minimal trauma, no risk of radiation and repeatable modality (18).

However, common complications following HIFU treatment have been reported, including pain in the treated area, subcutaneous edema, rib fractures and skin empyrosis (19). Due to the ultrasonic energy accumulation and deposition on the ribs, necrosis was previously reported to occur in all the layers of subcutaneous, costal and intercostal tissue following HIFU treatment. In that study, aseptic necrosis was prevented from spreading to the superficial skin by various treatments and nursing, thus avoiding skin ulceration and cellulitis of the necrotic subcutaneous tissue (17). In the present patient, the necrotic subcutaneous tissue was absorbed, which led to thinning of the chest wall structure and resulted in the occurrence of chest wall hernia. To date, chest wall hernia has not been reported in the literature as a complication of HIFU treatment.

In conclusion, despite certain complications associated with HIFU, this treatment may be an effective alternative modality for HCC patients who are not suitable for TACE, RFA or sorafenib treatment.

\section{Acknowledgements}

The authors would like to thank Dr Sonya Vargulick (Albany College of Pharmacy and Health Sciences, Albany, NY, USA) for editing the original manuscript.

\section{References}

1. Flores A and Marrero JA: Emerging trends in hepatocellular carcinoma: Focus on diagnosis and therapeutics. Clin Med Insights Oncol 8: 71-76, 2014.
2. Chang PE, Ong WC, Lui HF and Tan CK: Is theprognosis of young patients with hepatocellular carcinoma poorer than the prognosis of older patients? A comparative analysis of clinical characteristics, prognostic features, and survival outcome. J Gastroenterol 43: 881-888, 2008.

3. Rasool M, Rashid S, Arooj M, Ansari SA, Khan KM, Malik A, Naseer MI, Zahid S, Manan A, Asif M, et al: New possibilities in hepatocellular carcinoma treatment. Anticancer Res 34: 1563-1571, 2014.

4. Bruix J and Sherman M; American Association for the Study of Liver Diseases: Management of hepatocellular carcinoma: An update. Hepatology 53: 1020-1022, 2011.

5. Rust C and Gores GJ: Locoregional management of hepatocellular carcinoma. Surgical and ablation therapies. Clin Liver Dis 5: 161-173, 2001.

6. Brown MR, Farquhar-Smith P, Williams JE, ter Haar G and deSouza NM: The use of high-intensity focused ultrasound as a novel treatment for painful conditions - a description and narrative review of the literature. $\mathrm{Br} \mathrm{J}$ Anaesth 115: 520-530, 2015.

7. Zhang Y, Deng J, Feng J and Wu F: Enhancement of antitumor vaccine in ablated hepatocellular carcinoma by high-intensity focused ultrasound. World J Gastroenterol 16: 3584-3591, 2010.

8. Jiang L, Yan L, Wen T, Li B, Zeng Y, Yang J, Wang W, Xu M and $\mathrm{Wu} \mathrm{H}$ : Comparison of outcomes of hepatic resection and radiofrequency ablation for hepatocellular carcinoma patients with multifocal tumors meeting the Barcelona-Clinic Liver Cancer stage A classification. J Am Coll Surg 221: 951-961, 2015.

9. Zhong X, Lim EA, Hershman DL, Moinpour CM , Unger J, Lee SM, Zhong X, Lim EA, Hershman DL, Moinpour CM, et al: ReCAP: Identifying severe adverse event clusters using the National Cancer Institute's Common Terminology Criteria for Adverse Events. J Oncol Pract 12: 245-246, 2016.

10. Song T: Recent advances in surgical treatment of hepatocellular carcinoma. Drug Discov Ther 9: 319-330, 2015.

11. Hołówko W, Wróblewski T, Wojtaszek M, Grąt M, Kobryń K, Ziarkiewicz-Wróblewska B and Krawczyk M: Transarterial chemoembolization prior to liver transplantation in patients with hepatocellular carcinoma. Ann Transplant 20: 764-768, 2015.

12. Miyahara K, Nouso K and Yamamoto K: Chemotherapy for advanced hepatocellular carcinoma in the sorafenib age. World J Gastroenterol 20: 4151-4159, 2014.

13. Lee SH, Kim SU, Jang JW, Bae SH, Lee S, Kim BK, Park JY, Kim do Y, Ahn SH and Han KH: Use of transient elastography to predict de novo recurrence after radiofrequency ablation for hepatocellular carcinoma. Onco Targets Ther 8: 347-356, 2015.

14. Ten Eikelder HM, Bošnački D, Elevelt A, Donato K, Di Tullio A, Breuer BJ, van Wijk JH, van Dijk EV, Modena D, Yeo SY and Grüll H. Modelling the temperature evolution of bone under high intensity focused ultrasound. Phys Med Biol 61: $1810-1828,2016$.

15. de Greef M, Schubert G, Wijlemans JW, Koskela J, Bartels LW, Moonen CT and Ries M: Intercostal high intensity focused ultrasound for liver ablation: The influence of beam shaping on sonication efficacy and near-field risks. Med Phys 42: 4685-4697, 2015

16. Li CC, Wang YQ, Li YP and Li XL: High-intensity focused ultrasound for treatment of pancreatic cancer: A systematic review. J Evid Based Med 7: 270-281, 2014.

17. Wu F, Chen WZ, Bai J, Zou JZ, Wang ZL, Zhu H and Wang ZB: Pathological changes in human malignant carcinoma treated with high-intensity focused ultrasound. Ultrasound Med Biol 27: 1099-1106, 2001.

18. Blana A, Walter B, Rogenhofer S and Wieland WF: High-intensity focused ultrasound for the treatment of localized prostate cancer: 5-year experience. Urology 63: 297-300, 2004

19. Wang W, Wang Y, Wang T, Wang J, Wang L and Tang J: Safety and efficacy of US-guided high-intensity focused ultrasound for treatment of submucosal fibroids. Eur Radiol 22: $2553-2558,2012$. 